\title{
Endoplasmic Reticulum Stress and Heart Complication in Diabetes
}

\author{
Rida Malick and Souad Belmadani*
}

Department of Physiological Sciences, EVMS, Norfolk, Virginia 23501, USA

\begin{abstract}
Diabetes mellitus (DM), including type 1 and type 2, is a significant and substantially enhancing burden in adolescents and adults. Diabetic patients are at high risk for several cardiovascular disorders: coronary heart disease, stroke, peripheral arterial disease, cardiomyopathy, and congestive heart failure. Cardiovascular diseases are the most predominant cause of morbidity and mortality among diabetic patients. The pathophysiology of the diabetes-associated cardiovascular disease has not been completely elucidated. Therefore, there is a critical need for new therapies and clear strategies for research, prevention and treatment to stop the progression of the disease. The purpose of this mini-review is to discuss the recent literature linking heart, endoplasmic reticulum stress, and to address the potential role of endoplasmic reticulum stress in diabetic heart and potential therapies targeting its modulation.
\end{abstract}

Keywords: ER stress; Diabetes; UPR; Myocardium; Ischemia; Infarct

\section{Introduction}

Diabetes remains the $7^{\text {th }}$ leading cause of death in the United States and affects $9.3 \%$ of the American population with cardiovascular disease being the number one cause of mortality. According to a new analysis from the Center for Disease Control and Prevention, one-third of all adults will develop diabetes by 2050 [1].

Medical expenses for people with diabetes are about 2.3 times higher than would be without the disease. In 2012, the United States of America spent a total of $\$ 245$ billion dollars towards the cost of diagnosed diabetes with a $41 \%$ increase in expenditures from 2007 in which the total estimated cost was 174 billion dollars [1]. Thus, the increasing number of people with diabetes represents a significant burden on the economy and the public health in the United States.

As obesity rates rise worldwide, management of diabetes becomes increasingly important. Obesity is characterized with increased plasma leptin, TNF- $\alpha$ and non-esterified fatty acids that have been closely associated with, insulin resistance, type 2 diabetes, and cardiovascular disease. Excess nutrient intake is the primary cause of obesity. The endoplasmic reticulum (ER) stress appears to be the earliest consequence of nutrient excess and the reasons for the development of insulin resistance [2,3]. Thus, Ozcan et al. [4] demonstrated that obesity causes ER stress induction that in turn leads to a decrease in insulin receptor signaling. The authors found that the administration of chaperone proteins reduced ER stress and insulin resistance in mice. Moreover, ER stress is increased in subcutaneous fat of obese human subjects [5].

\section{Heart Failure and Diabetes}

Diabetes and heart failure (HF) are closely related and often coexist [6]. According to the Framingham Heart Study, the risk of HF was increased 2.4-fold in men and fivefold in women with diabetes [7]. In another study of 10,000 patients, HF was more frequent in diabetic patients; $11.8 \%$ versus $4.5 \%$ in non-diabetic patients [8]. The HF is a complex syndrome resulting from functional and structural disorders that affect the capacity of the heart to fill or eject blood. Complications associated with Type 2 diabetes such obesity, lifestyle and hypertension are well known to contribute to the cardiovascular disease in diabetic patients. HF accounts for $33 \%$ of diabetic hospitalizations [7,9].
Evidence from clinical, experimental, and epidemiological studies has shown that diabetes mellitus causes cardiac dysfunction independently of hypertension, coronary artery disease or any other cardiac diseases, known as diabetic cardiomyopathy (DCM) [10,11]. Diabetes is characterized by chronic low-grade inflammation and secretion/ activation of pro-inflammatory cytokines contributing to cardiac insulin resistance [12]. In addition to hyperglycemia, hyperlipidemia, and the activation of renin-angiotensin II- aldosterone system (RAAS), oxidative stress emerges as a major contributor to cardiac insulin resistance and subsequently damaging cardiac cells leading to DCM and $\mathrm{HF}[12,13]$.

Genetic predisposition to type 2 diabetes is associated with higher risk for coronary heart disease [14]. Even a slight increase in the glucose levels at the pre-diabetic stage can accelerate this progression if a genetic predisposition is present [14].

Intima-media thickness of the common carotid artery (IMT-CCA) is an early marker of atherosclerosis and associated with coronary heart disease [14]. IMT-CCA is increased in normal, overweight and obese glucose-tolerant patients with a family history of diabetes versus those with no family history of diabetes at all [14]. Mild insulin resistance in the pre-diabetic stage leads to endothelial dysfunction secondary to increased endothelial reactive oxygen species production [15]. Therefore the state of pre-diabetes, especially in those with a family history and increased IMT-CCA [14], may enhance the risk for cardiovascular disease.

The mechanisms underlying the diabetic heart complications are still not completely understood [11,16]. Recently, ER stress emerges as an important factor underlying heart function, DCM, and HF [16-19].

*Corresponding author: Dr. Souad Belmadani, Department of Physiologica Sciences, Eastern Virginia Medical School, Norfolk, Virginia 23501, USA, Tel: 757446-5880; E-mail: belmads@evms.edu

Received November 03, 2015; Accepted December 03, 2015; Published December 09, 2015

Citation: Malick R, Belmadani S (2015) Endoplasmic Reticulum Stress and Heart Complication in Diabetes. J Diabetes Metab 6: 630. doi:10.4172/2155 6156.1000630

Copyright: (C) 2015 Malick R, et al. This is an open-access article distributed under the terms of the Creative Commons Attribution License, which permits unrestricted use, distribution, and reproduction in any medium, provided the original author and source are credited. 


\section{Endoplasmic Reticulum (ER) Stress}

The ER is a multifunctional organelle with multiple vital functions such lipid synthesis, calcium homeostasis, protein folding, and maturation [20]. The ER responds to stress such ischemia, oxidative stress, and disturbance in calcium homeostasis by inhibiting protein translation and activating protein chaperones via a process called unfolded protein response (UPR). The UPR activates signaling pathways through 3 major ER resident proteins: the protein kinase R-like ER kinase (PERK), the inositol-requiring kinase-1 (IRE-1), and the activating transcription factor (ATF) 6 (Figure 1) [20]. In normal conditions, the ER luminal domain of PERK, ATF6 and IRE-
1 are bound to ER protein GRP78 and are inactive. When unfolded proteins accumulate in the $\mathrm{ER}, 78 \mathrm{kD}$ glucose-regulated protein GRP78 dissociates from the 3 ER-sensors and translocate to the misfolded proteins leading to the activation of the 3 ER sensor proteins. Initially, the UPR aims to compensate for damage and restore cellular homeostasis. However, once the UPR fails to control the level of unfolded and misfolded proteins in the ER, cellular dysfunction and apoptosis events occur (Figure 2). Abnormalities in the UPR have been involved in several diseases including cardiovascular diseases, [21] neurodegenerative diseases such as Parkinson's, Alzheimer's, prion disease [22] and cancer [23].

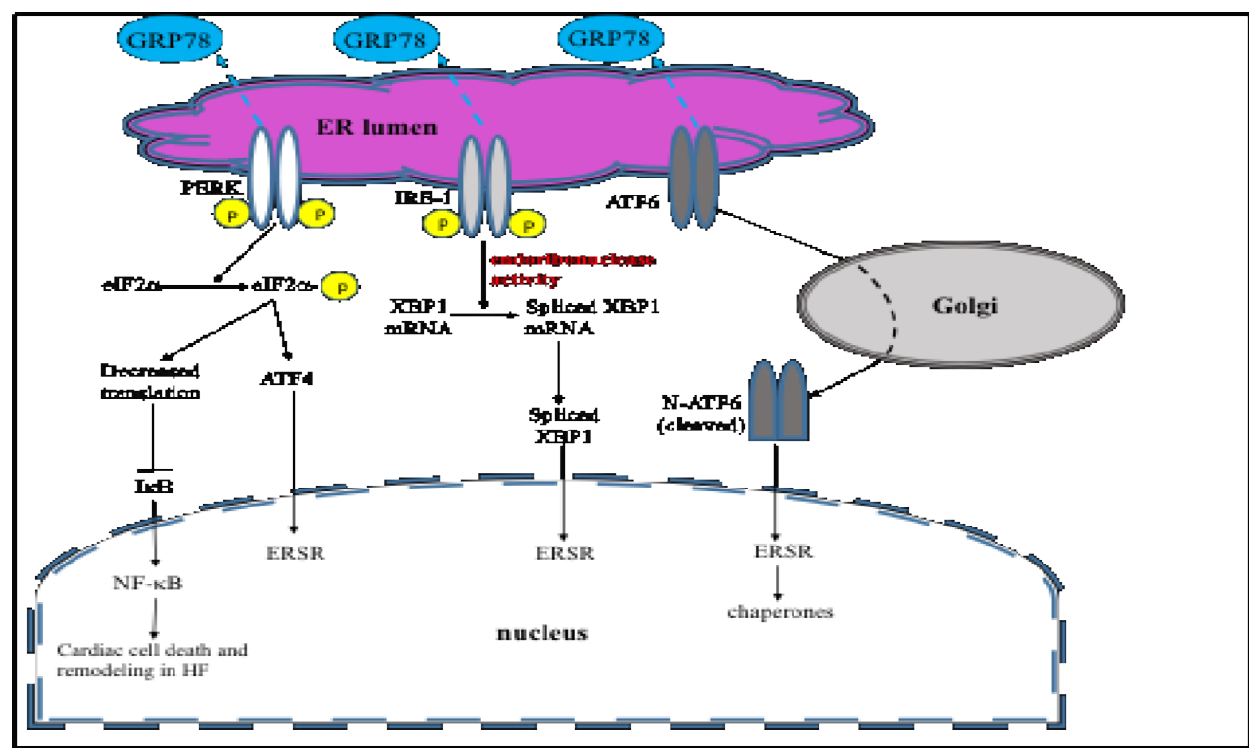

Figure 1: ER stress response activated by the three ER resident proteins - PERK, IRE-1, and ATF6.

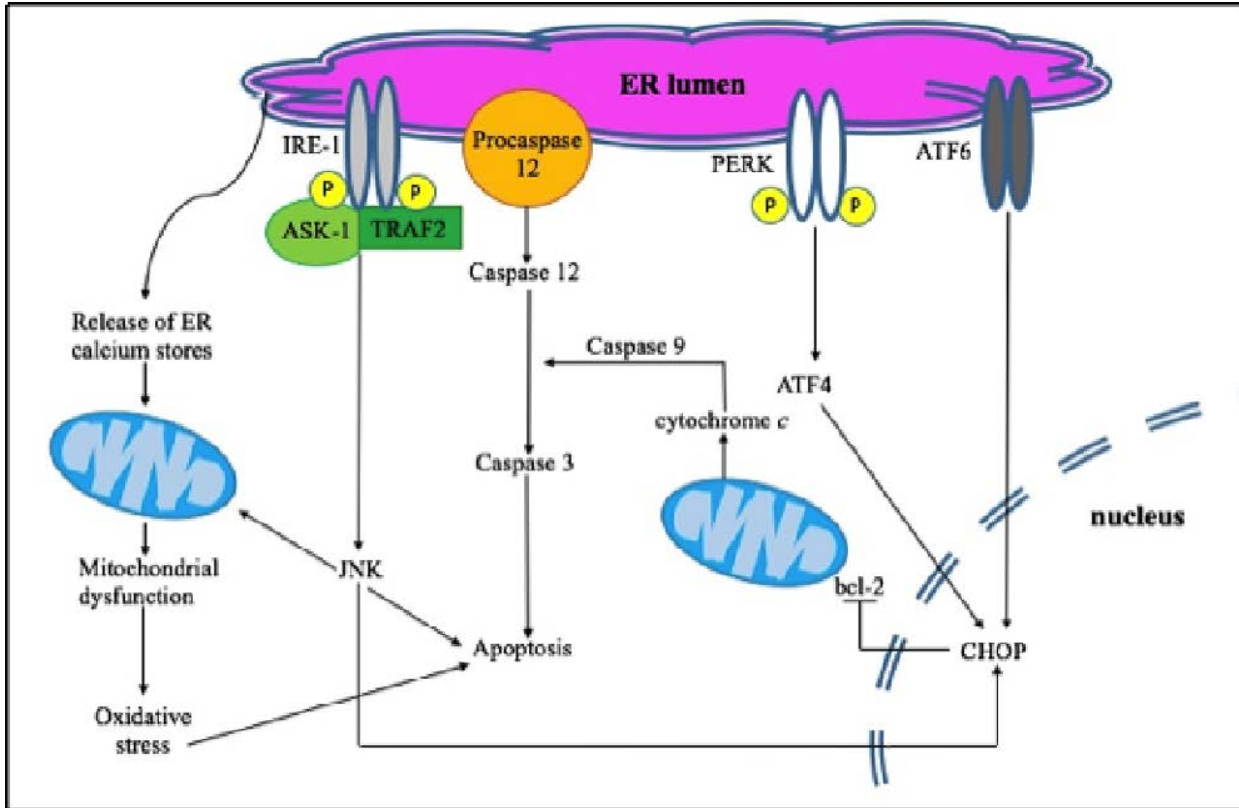

Figure 2: ERSR-induced cell death pathway. 


\section{Endoplasmic reticulum stress in heart}

The role of ER stress in the heart is still unclear. UPR is a favorable response to stress, but prolonged activation can be detrimental to the heart [19]. Hypoxia, pressure overload, and ischemia have been shown to disturb the ER and activate the UPR in the heart [24,25]. Mouse hearts subjected to ischemia-reperfusion injury exhibit an increase in ER stress protein GRP78 in the border zone of the injury, highlighting the importance of ER stress signaling in ischemia-reperfusion injury [26]. Moreover, other studies showed an induction of ER stress chaperone "GRP78" and the activation of ER stress involved in the development of ischemic heart disease in murine model expressing the inflammatory factor MCP-1 in the heart $[26,27]$. Glembotski and his team were the first to report the cardioprotective role of ATF6 after myocardial ischemia-reperfusion injury [28]. Using transgenic mice with a cardiac-restricted expression of the activated ER stress ATF6, the authors showed that ATF6 provides cardioprotection in a model of myocardial ischemia-reperfusion injury.

Other studies reported the UPR activation and ER stress induction in hypertrophic and failing hearts under experimental and clinical studies [29-31]. These studies were supported by the findings showing that the UPR was found in both hypertrophic and failing hearts [32]. The mRNA levels of ER stress components ATF4, CHOP, GRP78, and caspase 12 were up-regulated in a mouse model of heart failure $[33,34]$. Furthermore, the increased expression of ER stress-CHOP has been proposed as a key player in the transition from hypertrophy to heart failure [35]. Thus, the disruption of CHOP attenuates cardiac hypertrophy and dysfunction suggesting the potential of modulating CHOP in cardiac therapy. Recent studies revealed a crosstalk between ER stress and autophagy in the heart after ischemia-reperfusion injury highlighting the potential cardioprotective effect of ER stress inhibition [36]. Overall, these studies emphasize the complexity of the role played by the ER stress in the physiopathology of the heart. For instance, during myocardial infarction, the ATF6 seems to play a cardioprotective role [28], while IRE1a-ASK1 pathway-mediated ER stress appears to be detrimental [37]. The complexity relies on the fact that UPR signaling exerts dual biological function related to both survival and apoptotic pathways.

How the heart chooses between life or death once the UPR is activated and the ER stress is induced? How the ER stress is activated in cardiac physiology and cardiac pathology? How can we differentiate between the ER stress survival pathway and the ER stress detrimental pathway? How can we manipulate and activate the safe side of ER stress without stimulating the bad side? To answer these questions; future studies are needed to determine the exact role of each ER stress pathway in the heart and the contribution of theses pathways in myocardial infarction and heart failure [35].

\section{Endoplasmic reticulum (ER) stress in diabetic hearts}

Diabetes mellitus is one of the major risk factors for cardiac hypertrophy, inflammation, alterations in intracellular $\mathrm{Ca} 2+$ homeostasis, hyperglycemia, generation of reactive oxygen species, hyperinsulinemia, and insulin resistance, which can alter the homeostasis of the ER and induce the UPR [38]. In 1986, ultrastructure analysis showed a dilation of ER in diabetic myocardium suggesting the disturbance of ER and the induction of ER stress in diabetic hearts [39]. However, the relationship between ER stress and DCM is yet to be determined. In type 1 diabetic mouse model, ER stress in the heart was detected by the expression of ER chaperones, and apoptosis detected by CCAAT/enhancer-binding protein (C/EBP) homologous protein
(CHOP), cleaved caspase-3, and caspase-12 [40]. In diabetic mice, cardiac apoptosis was significantly increased two weeks after diabetes onset associated with significant up-regulation of the ER chaperones such glucose-regulated protein GRP78 and GRP94, cleaved ATF6, and phosphorylated eIF2 $\alpha$. Interestingly, Apoptosis was detected in myocytes, endothelial cells, and fibroblasts of ventricular myocardial biopsies obtained from diabetic patients [41].

In 2006, Ozcan et al. [42] showed that chemical or pharmaceutical chaperones, such as the 4-phenyl butyric acid (PBA) and tauroursodeoxycholic acid (TUDCA) reduce ER stress in a mouse model of type 2 diabetes. Subsequently, the inhibition of ER stress by TUDCA normalizes GRP78, GRP94, and mitochondrial GSK-3 $\beta$ in a rat model of type 2 diabetes resulting in a significant prevention of diabetic cardiac damage [43]. These findings suggest the link between ER stress and apoptosis events that occur in the diabetic heart. Thus, in vivo mechanistic and preclinical studies are needed to address the contribution of each ER stress pathway in the diabetic heart. Using various models of diabetes and different pathological states of cardiovascular system will enable us to determine whether manipulating ER stress can prevent diabetic cardiomyopathy in T2D.

\section{Diabetic cardiomyopathy a genetic understanding}

It is well established that a family history of diabetes can increase the risk of an individual to develop diabetes and cardiovascular disease [12]. Individuals with a first-degree relative with type 2 diabetes shows signs of insulin resistance before they even develop diabetes. Jermendy et al. [44] showed that while certain risk factors for diabetes are environmental, others such as weight circumference, serum creatinine, and blood pressure were associated with genetics and family history.

Epigenetics, including histone modification and methylation, and microRNAs (single- strand non coding RNAs) play a significant role in the development of cardiovascular disease and is a novel way to understand the pathophysiology of DCM [45]. External factors and stressors induce transcription factors to trigger gene expression resulting in changes in cardiac structure and remodeling [45]. Altered gene expression is present in DCM [45]. Hyperglycemia produces ROS that leads to increased expression of NF- $\mathrm{kB}$ subunit p65 [45], which is associated with cardiac cell death and cardiac remodeling in HF [46]. Hyperglycemia additionally induces changes in DNA methylation that is associated with diabetic HF [45].

miRNAs regulate gene expression at the post-transcriptional level by increasing degradation or decreasing translation of certain mRNAs. Alteration in the expression of relevant miRNAs is associated with DCM. miRNA-1, which represents 40 percent of the miRNA in the heart is linked to diabetes-induced cardiomyopathy [45]. The miRNA-133 is critical for cardiac hypertrophy and up-regulates MEF2A and MEF2C, two transcription factors involved in myocardial hypertrophy [47]. Additionally, the miRNA 133 controls connective tissue growth factor (CTGF), which induces fibrosis and thus contributes to DCM [45]. An extensive understanding of the role of genetics, epigenetics, and miRNAs in DCM can help us better understand the pathophysiology of DCM and acquire novel therapeutic targets.

Recently, a connection has been suggested between miRNAs and ER stress -dependent signaling [48]. In a clinical study, miR146a (involved in the regulation of inflammation) has been found to be down-regulated and negatively correlated with ER stress markers in type 2 diabetic patients [49]. The existence of such a relationship between ER stress signaling and miRNAs regulation offers a new way to regulate survival and death balance once the ER stress is activated. 


\section{Strategies and Associated Therapies}

Recent studies in the cardiovascular field reported that ER stress and UPR are highly activated in atherosclerosis, ischemic diseases, diabetes and heart failure. Therapies involving the modulation of the ER stress pathways show promising effects in the treatment of cardiovascular disease [50]. Two primary therapeutic strategies are proposed to target and modulate the ER stress response. The first approach consists in the activation of the adaptive ER stress response to restore cellular homeostasis and normalize cell function. The second procedure consists in inhibiting ER stress-induced apoptosis pathways to prevent cell death [50]. Studies on obesity and type 2 diabetes showed that Chemical chaperones such as a 4-phenyl butyric acid (PBA), and tauroursodeoxycholic acid (TUDCA) were able to alleviate the ER stress, correct hyperglycemia, normalize insulin sensitivity and restore Serca2a protein level in animal models $[42,50,51]$. These studies, open new opportunities for chemical chaperones therapies and clinical investigations for these molecules could be beneficial for cardiovascular complications in diabetes and obesity. Currently, TUDCA is used in 3 clinical trials on amyloid cardiomyopathy, the onset of type 1 diabetes and effect of ER stress on metabolic function.

Atorvastatin, a clinical statin drug, has been shown to improve left ventricular function and attenuate left ventricular remodeling in patients with heart failure [52]. In a rat model of heart failure, ER stress modulation was proposed as a mechanism by which atorvastatin protects the heart against heart failure [53]. In line with the statin drugs, Pravastatin was also shown to inhibit the UPR and prevents cardiac remodeling after pressure overload [54]. Valsartan, an Angiotensin II receptor type 1 antagonist, reduces ER stress response and prevents cardiac remodeling by blocking ER stress-CHOP and slowing down DCM [55].

AMP-activated protein kinase (AMPK) recognized as a cellular energy gauge have been extensively studied in the pathophysiology of the heart [56]. Dys-regulation of AMPK has been reported in humans and animal models of metabolic syndrome. AICAR and metformin, anti-diabetic drugs activate AMP Kinase (AMPK), reduce ER stress and slow the progression of HF [50]. AICAR additionally activates nuclear factor-E2-related factor (Nrf2) through AMPK independent pathways, which helps combat oxidative damage [57]. Increased expression of Nrf2 reduces cardiac hypertrophy, myocardial infarct, and the progression of HF [58]. However, AMPK and Nrf2 pathways show convergence as well [59]. Therapeutic targets that activate AMPK and Nrf2, as well as targets in UPR and apoptotic pathways, hold promise in the treatment of the diabetic heart. Therapeutic efforts aimed at oxidative stress also reduce the ER stress.

Thus, the ER stress appears to be a key player in cardiovascular complications related to type 2 diabetes. Also, a large number of drugs used in type 2 diabetes or heart failure involved ER stress modulation. Targeting ER stress pathways hold a great feature for diabetic patients. As the prevalence of diabetes rises yearly in the United States, it becomes significant to understand the relationship between diabetes and heart failure. Although the role of ER stress has been well established in diabetes, there is still much to learn about the contribution of ER stress in heart complication in diabetes. Through a better understanding of cardioprotective therapies tailored for diabetic hearts could be administered and potentially increase longevity and decrease morbidity and mortality due to diabetes.

\section{Summary and Perspectives}

ER stress has been involved in several cardiovascular complications and multiple studies linked ER stress to cell death in diabetes. The pathophysiological role of ER stress in diabetes in general and in diabetic cardiomyopathy specifically has progressed in recent years, but important issues are still unresolved. How cells choose between life and death once the ER stress is induced still remains unanswered. Activating the survival pathway and blocking cell death pathway induced by ER stress without altering autophagy is a significant challenge. Indeed, with the discovery of miRNAs and their potential connection with the ER stress markers, open new avenues. Depending on the nature of miRNAs and its up- or down-regulation, the impact on cell fate can be significantly modulated, leading to either prosurvival or pro-apoptotic effects. More basic research, clinical studies, and relevant pathological models are needed to alleviate the effects of ER stress in cardiovascular complications in diabetes. Understanding the cross-talk between the different ER stress signaling members and to be able to pharmacologically manipulate the UPR to favor pro-death or pro-survival pathway might provide a new treatment for cardiovascular complications in diabetes.

\section{References}

1. Roger VL, Go AS, Lloyd-Jones DM, Benjamin EJ, Berry JD, et al. (2012) Heart disease and stroke statistics--2012 update: a report from the American Heart Association. Circulation 125: e2-2e220.

2. Boden G (2009) Endoplasmic reticulum stress: another link between obesity and insulin resistance/inflammation? Diabetes 58: 518-519.

3. Leong KS, Wilding JP (1999) Obesity and diabetes. Baillieres Best Pract Res Clin Endocrinol Metab 13: 221-237.

4. Ozcan U, Cao Q, Yilmaz E, Lee AH, Iwakoshi NN, et al. (2004) Endoplasmic reticulum stress links obesity, insulin action, and type 2 diabetes. Science 306 457-461.

5. Boden G, Duan X, Homko C, Molina EJ, Song W, et al. (2008) Increase in endoplasmic reticulum stress-related proteins and genes in adipose tissue of obese, insulin-resistant individuals. Diabetes 57: 2438-2444.

6. Kannel WB, Hjortland M, Castelli WP (1974) Role of diabetes in congestive heart failure: the Framingham study. Am J Cardiol 34: 29-34.

7. Bell DS (2003) Heart failure: the frequent, forgotten, and often fatal complication of diabetes. Diabetes Care 26: 2433-2441.

8. Nichols GA, Gullion CM, Koro CE, Ephross SA, Brown JB (2004) The incidence of congestive heart failure in type 2 diabetes: an update. Diabetes Care 27: 1879-1884.

9. Matheus AS, Tannus LR, Cobas RA, Palma CC, Negrato CA, et al. (2013) Impact of diabetes on cardiovascular disease: an update. Int J Hypertens 2013 653789

10. Reis SE, Holubkov R, Edmundowicz D, McNamara DM, Zell KA, et al. (1997) Treatment of patients admitted to the hospital with congestive heart failure: specialty-related disparities in practice patterns and outcomes. J Am Coll Cardiol 30: 733-738.

11. de Simone G, Devereux RB, Chinali M, Lee ET, Galloway JM, et al. (2010) Diabetes and incident heart failure in hypertensive and normotensive participants of the Strong Heart Study. J Hypertens 28: 353-360.

12. Ciccone MM, Scicchitano P, Cameli M, Cecere A, Cortese F, et al. (2014) [Endothelial function in pre-diabetes, diabetes and diabetic cardiomyopathy: $A$ Review]. J Diabetes Metab 5: 364.

13. Leonardini A, Avogaro A (2013) Abnormalities of the cardiac stem and progenitor cell compartment in experimental and human diabetes. Arch Physio Biochem 119: 179-187.

14. Pannacciulli N, De Pergola G, Ciccone M, Rizzon P, Giorgino F, et al. (2003) Effect of family history of type 2 diabetes on the intima-media thickness of the common carotid artery in normal-weight, overweight, and obese glucosetolerant young adults. Diabetes Care 26: 1230-1234.

15. Duncan ER, Walker SJ, Ezzat VA, Wheatcroft SB, Li JM, et al. (2007) Accelerated endothelial dysfunction in mild prediabetic insulin resistance: the early role of reactive oxygen species. Am J Physiol Endocrinol Metab 293 E1311-1319. 
16. Yang L, Zhao D, Ren J, Yang J (2015) Endoplasmic reticulum stress and protein quality control in diabetic cardiomyopathy. Biochim Biophys Acta 1852: 209-218.

17. Boudina S, Abel ED (2007) Diabetic cardiomyopathy revisited. Circulation 115 : 3213-3223.

18. Xu J, Zhou Q, Xu W, Cai L (2012) Endoplasmic reticulum stress and diabetic cardiomyopathy. Exp Diabetes Res 2012: 827971.

19. Groenendyk J, Sreenivasaiah PK, Kim do H, Agellon LB, Michalak M (2010) Biology of endoplasmic reticulum stress in the heart. Circ Res 107: 1185-1197.

20. Xu C, Bailly-Maitre B, Reed JC (2005) Endoplasmic reticulum stress: cell life and death decisions. J Clin Invest 115: 2656-2664.

21. Hotamisligil GS (2010) Endoplasmic reticulum stress and the inflammatory basis of metabolic disease. Cell 140: 900-917.

22. Lindholm D, Wootz $H$, Korhonen $L$ (2006) ER stress and neurodegenerative diseases. Cell Death Differ 13: 385-392.

23. Zhao L, Ackerman SL (2006) Endoplasmic reticulum stress in health and disease. Curr Opin Cell Biol 18: 444-452.

24. Thuerauf DJ, Marcinko M, Gude N, Rubio M, Sussman MA, et al. (2006) Activation of the unfolded protein response in infarcted mouse heart and hypoxic cultured cardiac myocytes. Circ Res 99: 275-282.

25. Zhang PL, Lun M, Teng J, Huang J, Blasick TM, et al. (2004) Preinduced molecular chaperones in the endoplasmic reticulum protect cardiomyocytes from lethal injury. Ann Clin Lab Sci 34: 449-457.

26. Azfer A, Niu J, Rogers LM, Adamski FM, Kolattukudy PE (2006) Activation of endoplasmic reticulum stress response during the development of ischemic heart disease. Am J Physiol Heart Circ Physiol 291: H1411-1420.

27. Doroudgar S, Thuerauf DJ, Marcinko MC, Belmont PJ, Glembotski CC (2009) Ischemia activates the ATF6 branch of the endoplasmic reticulum stress response. J Biol Chem 284: 29735-29745.

28. Martindale JJ, Fernandez R, Thuerauf D, Whittaker R, Gude N, et al. (2006) Endoplasmic reticulum stress gene induction and protection from ischemia/ reperfusion injury in the hearts of transgenic mice with a tamoxifen-regulated form of ATF6. Circ Res 98: 1186-1193.

29. Ortega A, Rosello-Lleti E, Tarazon E, Molina-Navarro MM, Martinez-Dolz L, et al. (2014) Endoplasmic reticulum stress induces different molecular structural alterations in human dilated and ischemic cardiomyopathy. PLoS One 9: e107635.

30. Castillero E, Akashi H, Pendrak K, Yerebakan H, Najjar M, et al. (2015) Attenuation of the unfolded protein response and endoplasmic reticulum stress after mechanical unloading in dilated cardiomyopathy. Am J Physiol Heart Circ Physiol 309: H459-470.

31. Okada K, Minamino T, Tsukamoto Y, Liao Y, Tsukamoto O, et al. (2004) Prolonged endoplasmic reticulum stress in hypertrophic and failing heart after aortic constriction: possible contribution of endoplasmic reticulum stress to cardiac myocyte apoptosis. Circulation 110: 705-712.

32. Ceylan-Isik AF, Sreejayan N, Ren J (2011) Endoplasmic reticulum chaperon tauroursodeoxycholic acid alleviates obesity-induced myocardial contractile dysfunction. J Mol Cell Cardiol 50: 107-116.

33. Toko H, Takahashi H, Kayama Y, Okada S, Minamino T, et al. (2010) ATF6 is important under both pathological and physiological states in the heart. J Mo Cell Cardiol 49: 113-120

34. Liu Y, Wang J, Qi SY, Ru LS, Ding C, et al. (2014) Reduced endoplasmic reticulum stress might alter the course of heart failure via caspase-12 and JNK pathways. Can J Cardiol 30: 368-375.

35. Fu HY, Okada K, Liao Y, Tsukamoto O, Isomura T, et al. (2010) Ablation of $\mathrm{C} / \mathrm{EBP}$ homologous protein attenuates endoplasmic reticulum-mediated apoptosis and cardiac dysfunction induced by pressure overload. Circulation 122: 361-369.

36. Petrovski G, Das S, Juhasz B, Kertesz A, Tosaki A, et al. (2011) Cardioprotection by endoplasmic reticulum stress-induced autophagy. Antioxid Redox Signal 14 2191-2200.

37. Yamaguchi O, Higuchi Y, Hirotani S, Kashiwase K, Nakayama H, et al. (2003)
Targeted deletion of apoptosis signal-regulating kinase 1 attenuates left ventricular remodeling. Proc Natl Acad Sci U S A 100: 15883-15888.

38. Schilling JD, Mann DL (2012) Diabetic cardiomyopathy: bench to bedside. Heart Fail Clin 8: 619-631.

39. Jackson CV, McGrath GM, Tahiliani AG, Vadlamudi RV, McNeill JH (1985) A functional and ultrastructural analysis of experimental diabetic rat myocardium. Manifestation of a cardiomyopathy. Diabetes 34: 876-883.

40. Xu J, Wang G, Wang Y, Liu Q, Xu W, et al. (2009) Diabetes- and angiotensin II-induced cardiac endoplasmic reticulum stress and cell death: metallothionein protection. J Cell Mol Med 13: 1499-1512.

41. Frustaci A, Kajstura J, Chimenti C, Jakoniuk I, Leri A, et al. (2000) Myocardial cell death in human diabetes. Circ Res 87: 1123-1132.

42. Ozcan U, Yilmaz E, Ozcan L, Furuhashi M, Vaillancourt E, et al. (2006) Chemical chaperones reduce ER stress and restore glucose homeostasis in a mouse model of type 2 diabetes. Science 313: 1137-1140.

43. Miki T, Miura T, Hotta H, Tanno M, Yano T, et al. (2009) Endoplasmic reticulum stress in diabetic hearts abolishes erythropoietin-induced myocardial protection by impairment of phospho-glycogen synthase kinase-3beta-mediated suppression of mitochondrial permeability transition. Diabetes 58: 2863-2872.

44. Jermendy G, Horváth T, Littvay L, Steinbach R, Jermendy AL, et al. (2011) Effect of genetic and environmental influences on cardiometabolic risk factors: a twin study. Cardiovasc Diabetol 10: 96.

45. Asrih M, Steffens S (2013) Emerging role of epigenetics and miRNA in diabetic cardiomyopathy. Cardiovasc Pathol 22: 117-125

46. Hamid T, Guo SZ, Kingery JR, Xiang X, Dawn B, et al. (2011) Cardiomyocyte NF-1 ${ }^{\circ} \mathrm{B}$ p65 promotes adverse remodelling, apoptosis, and endoplasmic reticulum stress in heart failure. Cardiovasc Res 89: 129-138.

47. Zhou Q, Lv D, Chen P, Xu T, Fu S, et al. (2014) MicroRNAs in diabetic cardiomyopathy and clinical perspectives. Front Genet 5: 185.

48. Maurel M, Chevet E (2013) Endoplasmic reticulum stress signaling: the microRNA connection. Am J Physiol Cell Physiol 304: C1117-1126.

49. Lenin R, Sankaramoorthy A, Mohan V, Balasubramanyam M (2015) Altered immunometabolism at the interface of increased endoplasmic reticulum (ER) stress in patients with type 2 diabetes. J Leukoc Biol 98: 615-622.

50. Minamino T, Komuro I, Kitakaze M (2010) Endoplasmic reticulum stress as a therapeutic target in cardiovascular disease. Circ Res 107: 1071-1082.

51. Takada A, Miki T, Kuno A, Kouzu H, Sunaga D, et al. (2012) Role of ER stress in ventricular contractile dysfunction in type 2 diabetes. PLoS One 7: e39893.

52. Sola S, Mir MQ, Lerakis S, Tandon N, Khan BV (2006) Atorvastatin improves left ventricular systolic function and serum markers of inflammation in nonischemic heart failure. J Am Coll Cardiol 47: 332-337.

53. Song XJ, Yang CY, Liu B, Wei Q, Korkor MT, et al. (2011) Atorvastatin inhibits myocardial cell apoptosis in a rat model with post-myocardial infarction heart failure by downregulating ER stress response. Int J Med Sci 8: 564-572.

54. Zhao H, Liao Y, Minamino T, Asano Y, Asakura M, et al. (2008) Inhibition of cardiac remodeling by pravastatin is associated with amelioration of endoplasmic reticulum stress. Hypertens Res 31: 1977-1987.

55. Wu T, Dong Z, Geng J, Sun Y, Liu G, et al. (2011) Valsartan protects agains ER stress-induced myocardial apoptosis via CHOP/Puma signaling pathway in streptozotocin-induced diabetic rats. Eur J Pharm Sci 42: 496-502.

56. Qi D, Young LH (2015) AMPK: energy sensor and survival mechanism in the ischemic heart. Trends Endocrinol Metab 26: 422-429.

57. Sid B, Glorieux C, Valenzuela M, Rommelaere G, Najimi M, et al. (2014) AICAR induces Nrf2 activation by an AMPK-independent mechanism in hepatocarcinoma cells. Biochem Pharmacol 91: 168-180.

58. Cominacini L, Mozzini C, Garbin U, Pasini A, Stranieri C, et al. (2015) Endoplasmic reticulum stress and Nrf2 signaling in cardiovascular diseases. Free Radic Biol Med 88: 233-242.

59. Mo C, Wang L, Zhang J, Numazawa S, Tang H, et al. (2014) The crosstalk between Nrf2 and AMPK signal pathways is important for the anti-inflammatory effect of berberine in LPS-stimulated macrophages and endotoxin-shocked mice. Antioxid Redox Signal 20: 574-588. 